\title{
Is Radioactive Decay Really Exponential?
}

\author{
Philip J. Aston* \\ Department of Mathematics \\ University of Surrey \\ Guildford \\ Surrey GU2 7XH \\ $\mathrm{UK}$
}

April 27, 2012

\begin{abstract}
Radioactive decay of an unstable isotope is widely believed to be exponential. This view is supported by experiments on rapidly decaying isotopes but is more difficult to verify for slowly decaying isotopes. The decay of ${ }^{14} \mathrm{C}$ can be calibrated over a period of 12,550 years by comparing radiocarbon dates with dates obtained from dendrochronology. It is well known that this approach shows that radiocarbon dates of over 3,000 years are in error, which is generally attributed to past variation in atmospheric levels of ${ }^{14} \mathrm{C}$. We note that predicted atmospheric variation (assuming exponential decay) does not agree with results from modelling, and that theoretical quantum mechanics does not predict exact exponential decay. We give mathematical arguments that non-exponential decay should be expected for slowly decaying isotopes and explore the consequences of non-exponential decay. We propose an experimental test of this prediction of non-exponential decay for ${ }^{14} \mathrm{C}$. If confirmed, a foundation stone of current dating methods will have been removed, requiring a radical reappraisal both of radioisotope dating methods and of currently predicted dates obtained using these methods.
\end{abstract}

\section{Introduction}

Radioactive decay is almost universally believed to satisfy the exponential decay law over many half lives and in particular cases where it has been tested [1, 2] it has been found to accurately represent the decay of an unstable isotope. However, as nuclear physics as a discipline is little over a century old, these experiments have necessarily been restricted to rapidly decaying isotopes. This principle of exponential decay is then assumed to hold in many other situations where there is no direct experimental verification, notably for slowly decaying isotopes which are widely used for dating objects ranging from a few hundred years old, to billions of years old. Newton summarised this situation as follows: 'Although in some instances the exponential law has been experimentally well verified over many lifetimes, it has certainly not been checked in many cases in which it is nevertheless assumed to be valid' [3, p608].

So is there any experimental or theoretical evidence that can be used to verify, or disprove, this widely accepted assumption for slowly decaying isotopes?

*Email: P.Aston@surrey.ac.uk 
With regard to experimental evidence, we consider in detail the decay of ${ }^{14} \mathrm{C}$ which is the basis of radiocarbon dating. We propose that non-exponential decay may be a contributing factor in the known discrepancy in radiocarbon dates of over 3,000 years. We also consider the theoretical aspects of radioactive decay as described by quantum mechanics, and again present arguments for non-exponential decay for slowly decaying isotopes. We then consider the consequences if non-exponential decay is assumed and conclude with a proposal for an experimental test of non-exponential decay.

\section{Radiocarbon dating and calibration}

The radioisotope ${ }^{14} \mathrm{C}$ is the basis for radiocarbon dating in which it is assumed that living organisms have a ${ }^{14} \mathrm{C} /{ }^{12} \mathrm{C}$ ratio which is the same as that in the atmosphere and that this atmospheric ratio has been constant in the past. The interaction between the organism and the atmosphere stops at death, and the ${ }^{14} \mathrm{C}$ that remains in the organism decays. By measuring the ${ }^{14} \mathrm{C} /{ }^{12} \mathrm{C}$ ratio directly (using Accelerator Mass Spectrometry (AMS)) or the rate of decay of ${ }^{14} \mathrm{C}$ (using radiometric methods), the time since death can be estimated. Clearly there is another assumption underlying this process, namely that the decay of ${ }^{14} \mathrm{C}$ is exactly exponential for all time. The half-life for ${ }^{14} \mathrm{C}$ was initially found to be $5,568 \pm 30 \mathrm{yr}$ (the Libby half-life) but was later changed to 5,730 $\pm 40 \mathrm{yr}$ (the Cambridge half life) [4]. The Libby half-life is still used by convention in calculating ${ }^{14} \mathrm{C}$ ages [4, 5, 7].

Radiocarbon dates can be calibrated using dendrochronology in which the age of a sequence of trees of increasing age can be determined very accurately by counting the growth rings and cross-dating different samples of overlapping age [4]. There can be occasional problems with missing rings or double rings produced in a single year, but with careful analysis and checking of several samples the errors in this process are very small ('on the order of 1 yr' [8]). The longest continuous tree-ring chronology of 12,593 years is based on German oak and pine trees, supplemented by pines from Switzerland [9, 10]. This approach is unique in providing accurate, absolute dates over a period of many thousands of years.

The dates of a tree sample obtained using dendrochronology and by radiocarbon dating are found to be very similar over the initial 3,000 year period, but for older trees there is a significant difference between these two dates of up to $15 \%$. It is assumed that the date obtained from dendrochronology is the more accurate one and so these two dating methods can be used to generate a calibration curve for converting radiocarbon dates to calendar dates. The currently accepted calibration curve is INTCAL09 [11] which is based on dendrochronology for the first 12,550 years and extends back to 50,000 years using other methods. We note that radiocarbon dates have units $\mathrm{BP}$ (before present, where the present is the base year of 1950) or $\mathrm{kBP}$, while calibrated dates obtained from the calibration curve have units cal BP or cal $\mathrm{kBP}$.

While the use of the calibration curve may have resolved the issue for the practitioner, who now has a valuable and accurate dating tool, for the theorist it leaves the obvious question as to what is the cause of this discrepancy in the radiocarbon dates?

\section{Atmospherics}

The discrepancy in the calibration curve is generally attributed to past atmospheric variation in the ${ }^{14} \mathrm{C} /{ }^{12} \mathrm{C}$ ratio, which contradicts the assumption made in the dating process that the ratio was constant in the past. Four factors are considered to affect this atmospheric ratio, namely primary cosmic ray flux, strength of the solar electromagnetic field, terrestrial magnetic field 
intensity and the structure of the carbon cycle [4, 12, 13]. The combined effect of these factors over the last 12,550 years is not known and since we do not have an independent method of measuring the atmospheric ratio over many thousands of years, it is not possible to correlate the variation in the atmospheric ratio with the discrepancy in the calibration curve. However, this is a significant assumption made in the carbon dating process which is known not to hold and so is generally considered to be the sole cause of the discrepancy in the radiocarbon dates.

If the decay of ${ }^{14} \mathrm{C}$ is assumed to be exponential, then the discrepancy in the calibration curve can be used to predict the variation $\Delta^{14} \mathrm{C}$ of the atmospheric ratio in the past [14]. Data from a stalagmite [13] and from marine sediments [15] have been used to construct a record of $\Delta^{14} \mathrm{C}$ going back to 45 or 50 cal $\mathrm{kBP}$ respectively. These records show much larger variations than are found over the 12.55 cal kBP period that we have considered. Both studies also used various box models of the global carbon cycle, which typically did not achieve levels of $\Delta^{14} \mathrm{C}$ as high as those derived from the data unless extreme values of the parameters were used. Chiu et al. [16] analysed these models and stated that 'there is no commonly accepted explanation for the high atmospheric $\Delta^{14} \mathrm{C}$ values recorded in most archives'. They also concluded that 'the discrepancies between measured $\Delta^{14} \mathrm{C}$ and modelled $\Delta^{14} \mathrm{C}$ remain unresolved'. They noted that one way to reduce the predicted high levels of $\Delta^{14} \mathrm{C}$ would be to increase the half life of ${ }^{14} \mathrm{C}$. A half life of 6,030 years gives results that are 'entirely consistent with the Beck et al. (2001) model (A) prediction' [16], which corresponds to an increase in the half life of over $5 \%$. However, recent experimental results have proposed that the half life should be decreased by $2 \pm 1 \%$ [17], which would of course increase the predicted $\Delta^{14} \mathrm{C}$ values even further, and so there is no justification for the increase in the half life that is required to reduce the high $\Delta^{14} \mathrm{C}$ values.

While the date range used by these authors is way beyond the range that we are considering, these studies suggest that variation in the atmospheric ratio may be insufficient on its own to explain the discrepancy in the calibration curve. However, allowing for non-exponential decay as well as atmospheric variation would mean that the predicted $\Delta^{14} \mathrm{C}$ curve could be reduced to be more in line with the predictions obtained from the modelling. So is it possible that some of the discrepancy in the radiocarbon dates could be due to non-exponential decay of ${ }^{14} \mathrm{C}$ ?

\section{A statistical perspective}

Before considering the possibility of non-exponential decay, we first review the common simple derivation of exponential decay.

Radioactive decay occurs when an unstable atomic nucleus spontaneously emits energy and matter, often transforming into a new element in the process. The unstable ${ }^{14} \mathrm{C}$ nucleus can undergo radioactive beta decay in which an electron and an anti-neutrino are emitted and the ${ }^{14} \mathrm{C}$ decays to the stable isotope ${ }^{14} \mathrm{~N}$. This decay process is assumed to be stochastic at the atomic level and hence unpredictable, but the average rate of decay for a large number of atoms is often highly predictable. Assuming that each atom is equally likely to decay at any time and that the probability of decay is independent of the age of the atom leads to a Poisson process [18] which can be described in terms of the differential equation

$$
\dot{A}=-\alpha A, \quad A(0)=A_{0},
$$

where $A(t)$ is the ${ }^{14} \mathrm{C} /{ }^{12} \mathrm{C}$ ratio at time $t$ with initial value $A_{0}$ at time $t=0$, which corresponds to the atmospheric ratio, $\alpha$ is the decay constant which determines the rate of decay and $\dot{A}=\mathrm{d} A / \mathrm{d} t$. The solution of equation (1) is

$$
A(t)=A_{0} e^{-\alpha t},
$$


which gives the expected exponential decay.

We note that this derivation of exponential decay is based on a simple statistical argument and makes no attempt to understand the mechanism that causes the decay. It has been noted that 'the exponential law... is experimentally verified in some cases to an astonishing degree, much more so than one has a right to expect considering the rather flimsy basis on which it is usually derived' [3, p594]. So what is the correct theoretical basis for understanding radioactive decay?

\section{A quantum mechanical perspective}

The theory of quantum mechanics describes the process of radioactive decay in terms of solutions of the Schrödinger equation [19, 20]. This theory highlights one of the deficiencies of the simple equation (11), namely that it does not contain history-dependent terms, as are contained in the equation for the probability amplitude derived from the theory of quantum mechanics [19, 21].

The general solution of the Schrödinger equation using first order perturbation theory does not give the probability of non-decay as a simple exponential. However, if some approximations are used, known as the Fermi Golden Rule [19, 20], then this probability amplitude becomes exponential. Thus, "the exponential decay law, for which we have so much empirical support in radioactive decay processes, is not a rigorous consequence of quantum mechanics, but the result of somewhat delicate approximations' [20, p513].

The theory of quantum mechanics predicts variations from pure exponential decay at very short times and at very long times [21. However, these deviations occur in regimes that are currently beyond the reach of experimental verification [2].

Thus, there is no theoretical basis from quantum mechanics for exponential decay in all circumstances. We might therefore ask why the theoretical possibility of non-exponential decay has not been observed experimentally.

\section{A mathematical perspective}

One important mathematical property of the differential equation (11) is that it is linear. Ian Stewart observed that 'today's science shows that nature is relentlessly nonlinear' [22, p83]. This does not mean that there is no room for linear equations but if the world is essentially nonlinear, then linearity is a special case and as such must be justified for a particular problem. So could this linear equation in reality be an approximation of a nonlinear equation? And what difference would it make if this equation was actually nonlinear?

To address these questions, we note that in the context of radioactive decay, there are two quantities that can be used to distinguish four scenarios namely (i) the rate of decay and (ii) the timescale (relative to the half life) over which measurements are made. This is illustrated in fig. 1. We discuss the terms "rapid decay" and "slow decay" in more detail later.

We first consider the short time case. Plotting $A(t)$ on a log scale results in a straight line graph if $A(t)$ is exponential. On a timescale that is small relative to the half life, only a short section of the line is observed and this may be approximately straight, even if it is not straight over a much longer timescale, so that decay, when considered over a short time period, appears to be exponential. This covers the two possibilities in the bottom row of fig. 1,

In quantum mechanics, the decay of an unstable state is described by the Schrödinger equation, from which a convolution differential equation can be derived for a complex transition amplitude $z(t)$ from the initial unstable state to itself. The probability of finding the system 


\begin{tabular}{c|c|c|}
\cline { 2 - 3 } $\begin{array}{c}\text { Long time } \\
(\alpha t \text { large })\end{array}$ & $\begin{array}{c}\text { Exponential } \\
\text { decay } \\
\text { (Observed })\end{array}$ & $\begin{array}{c}\text { Non-exponential } \\
\text { decay? } \\
\text { (Not observed })\end{array}$ \\
\cline { 2 - 3 } $\begin{array}{c}\text { Short time } \\
(\alpha t \text { small })\end{array}$ & $\begin{array}{c}\text { Exponential } \\
\text { decay } \\
(\text { Observed })\end{array}$ & $\begin{array}{c}\text { Exponential } \\
\text { decay } \\
(\text { Observed })\end{array}$ \\
\cline { 2 - 3 } & $\begin{array}{c}\text { Rapid decay } \\
(\alpha \text { large })\end{array}$ & $\begin{array}{c}\text { Slow decay } \\
(\alpha \text { small })\end{array}$
\end{tabular}

Figure 1: The type of decay that is likely in four different cases.

still in the initial state at time $t$ is $|z(t)|^{2}$, which is the quantity that is usually assumed to decay exponentially [20, 21].

The Schrödinger equation is linear, which follows from the Principle of Superposition [20], as is the derived convolution differential equation for $z(t)$. However, if we work with the two real variables $A=|z|^{2}$ and $\theta=\arg (z)$, then the differential equations in these two variables are nonlinear. Scaling $A$ by $1 / A_{0}$ ensures that it satisfies the initial condition $A(0)=1$. The equation for $A$ can then be expressed in the form

$$
\dot{A}=-\alpha A+g(A, \theta, t),
$$

where the nonlinear function $g$ involves an integral over past time and satisfies $g(0, \theta, t)=0$. We are not concerned with the equation for the second variable $\theta$.

We now define rapid decay to be when $\alpha$ is large compared to the nonlinear term $g(A, \theta, t)$ and slow decay to be when the two terms are of comparable magnitude.

1. If decay is rapid, then the nonlinear term in equation (2) will be small compared to the linear term resulting in decay that is very close to exponential for a long time. This covers the top left box of fig. 1.

2. If decay is slow, then the linear and nonlinear terms will be more comparable in magnitude, and the nonlinear term will then play an important part in the decay dynamics, resulting in non-exponential decay. This is the remaining combination in fig. 1.

The above discussion contains a number of assumptions which cannot easily be verified. However, it is undoubtedly true that accurate measurements have been made in three cases, as also indicated in fig. 1, with all such measurements strongly supporting the assumption of exponential decay. For example, the decay of ${ }^{56} \mathrm{Mn}$, which has a half life of $2.5785 \mathrm{~h}$, has been measured experimentally up to 45 half lives in order to search for non-exponential effects, but none were found [2]. In the fourth case, it has not been possible to accurately measure slow decay over long time periods, which is precisely the case where it is hard to justify the assumption of a linear equation giving rise to exact exponential decay.

Thus, it is possible that the slow decay of ${ }^{14} \mathrm{C}$ could be non-exponential, and consequently that this non-exponential decay is a contributing factor to the discrepancy in the radiocarbon dates described above. 


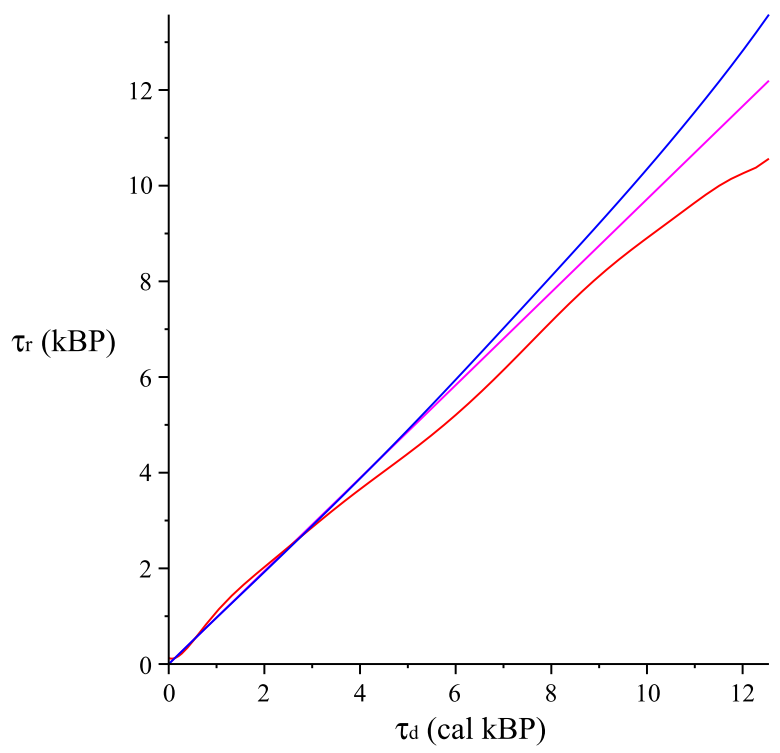

Figure 2: The calibration curves $C_{\beta}$ (red) and $C_{\mathrm{AMS}}$ (blue) together with the line $\tau_{r}=\left(\alpha_{C} / \alpha_{L}\right) \tau_{d}$ (magenta).

\section{$7 \quad$ What if?}

We have thus far made a case that variation in the atmospheric ratio may be insufficient on its own to explain the discrepancy in the calibration curve, and that the possibility of nonexponential decay should also be considered. We therefore now assume that the discrepancy in the calibration curve is a combination of atmospheric variation and non-exponential decay. Of course our big problem, having made this assumption, is that we do not know how much each of these two factors contributes to the discrepancy. There are two extremes that could be considered, namely (i) that the discrepancy is entirely due to atmospheric variation (and hence that decay is exponential) or (ii) that there is no atmospheric variation and the discrepancy is entirely due to non-exponential decay. Case (i) is usually assumed to apply, and the consequences of this assumption have been studied, resulting in the $\Delta^{14} \mathrm{C}$ curve which we discussed above. We now make the alternative extreme assumption described by case (ii), in order to consider the consequences of this assumption, whilst recognising that this does not occur in practice.

Before continuing, we digress briefly to consider the INTCAL09 dataset [1] which consists of an underlying curve with superimposed small oscillations, known as "wiggles". A close correspondence between these wiggles and solar activity related to sunspots over a period of 400 years has been observed [12, 23]. Thus, we ignore the wiggles and extract the underlying trend in the data. Let $\tau_{r}$ and $\tau_{d}$ be the dates of a tree sample obtained using radiocarbon dating and dendrochronology respectively. We fit the data with a polynomial of degree 12 which we denote by $\tau_{r}=C_{\beta}\left(\tau_{d}\right)$. This smoothed curve is shown in fig. 2 .

There are two methods used for carbon dating, namely radiometric methods ( $\beta$-counting), which measure the rate of decay, and AMS, which measures the ${ }^{14} \mathrm{C} /{ }^{12} \mathrm{C}$ ratio directly. $\mathrm{A}$ consequence of the assumption in case (ii) is that these two methods will give different predicted dates for a given sample. To quantify this difference, let $Y(t)$ be the (non-exponential) decay profile of the ${ }^{14} \mathrm{C} /{ }^{12} \mathrm{C}$ ratio which satisfies $Y(0)=A_{0}$, where $A_{0}$ is the (assumed constant) value of the atmospheric ratio. We also define a non-dimensional decay function $y(t)=Y(t) / A_{0}$ which is the proportion of the original ${ }^{14} \mathrm{C}$ that remains at time $t$, and satisfies the initial condition 
$y(0)=1$.

With radiometric methods, the sample activity, or rate of decay, is measured, normalised to take account of isotopic fractionation, and adjusted to the 1950 level. This normalised sample activity, denoted by $A_{\mathrm{SN}}$, is compared with the similarly normalised activity of an oxalic acid standard, denoted by $A_{\mathrm{ON}}$, which is considered to be the same as the activity of the atmospheric carbon. The radiocarbon age $\tau_{r}(\mathrm{BP})$ of the sample is then given by [5]

$$
\tau_{r}=-\frac{1}{\alpha_{L}} \ln \left(\frac{A_{\mathrm{SN}}}{A_{\mathrm{ON}}}\right),
$$

where $\alpha_{L}=\ln 2 / 5568 \mathrm{yr}^{-1}$, which involves the Libby half life.

If the decay curve is given by $Y(t)$, then the activity of the sample at time $t_{0}$ is $A_{\mathrm{SN}}=-\dot{Y}\left(t_{0}\right)$. The activity of the standard, assuming that decay is exponential initially, is $A_{\mathrm{ON}}=\alpha_{C} A_{0}$, where $\alpha_{C}=\ln 2 / 5730 \mathrm{yr}^{-1}$, which is the decay constant based on the more accurate Cambridge half-life. Substituting for $A_{\mathrm{SN}}$ and $A_{\mathrm{ON}}$ in (3) then gives

$$
\tau_{r}=-\frac{1}{\alpha_{L}} \ln \left(-\frac{\dot{Y}\left(t_{0}\right)}{\alpha_{C} A_{0}}\right)=-\frac{1}{\alpha_{L}} \ln \left(-\frac{\dot{y}\left(t_{0}\right)}{\alpha_{C}}\right) .
$$

Rearranging equation (4) gives

$$
\dot{y}\left(t_{0}\right)=-\alpha_{C} e^{-\alpha_{L} \tau_{r}} .
$$

The calibration curve INTCAL09 in the range of interest of 0-12.55 cal kBP was derived almost exclusively using radiometric methods. Using the smoothed calibration curve we therefore have that $\tau_{r}=C_{\beta}\left(\tau_{d}\right)$ where $\tau_{d}$ is the true age of the sample obtained by dendrochronology. This is the same as the time $t_{0}$, and so we have

$$
\dot{y}\left(t_{0}\right)=-\alpha_{C} e^{-\alpha_{L} C_{\beta}\left(t_{0}\right)} .
$$

This equation holds for all values of $t_{0}$, and so we replace it with $t$. Integrating and using the initial condition $y(0)=1$ then gives

$$
y(t)=1-\alpha_{C} \int_{0}^{t} e^{-\alpha_{L} C_{\beta}(s)} \mathrm{d} s .
$$

This function is compared with the corresponding exponential decay curve $e^{-\alpha_{C} t}$ in fig. 3, from which it can be observed that there is very good agreement between the two curves initially with more of a difference developing after approximately 6,000 years.

The ratio of the function $y(t)$ and the exponential $e^{-\alpha_{C} t}$ shows small amplitude oscillations initially (see fig. (4). These arise due to the fact that the calibration curve initially oscillates also (see fig. 2). In a simple tunnelling problem, the survival probability was found to be nonexponential, and also showed oscillations [6], although the amplitude was much larger than those shown in fig. 4 .

We note that if it is assumed that decay is exponential, then substituting $y\left(t_{0}\right)=e^{-\alpha_{C} t_{0}}$ into (4) and replacing $t_{0}$ with the dendrochronologically determined age $\tau_{d}$ gives

$$
\tau_{r}=\frac{\alpha_{C}}{\alpha_{L}} \tau_{d}=0.9717 \tau_{d},
$$

which is slightly lower than the line $\tau_{r}=\tau_{d}$. The reason for this is the historic use of $\alpha_{L}$ rather than $\alpha_{C}$ in (3). 


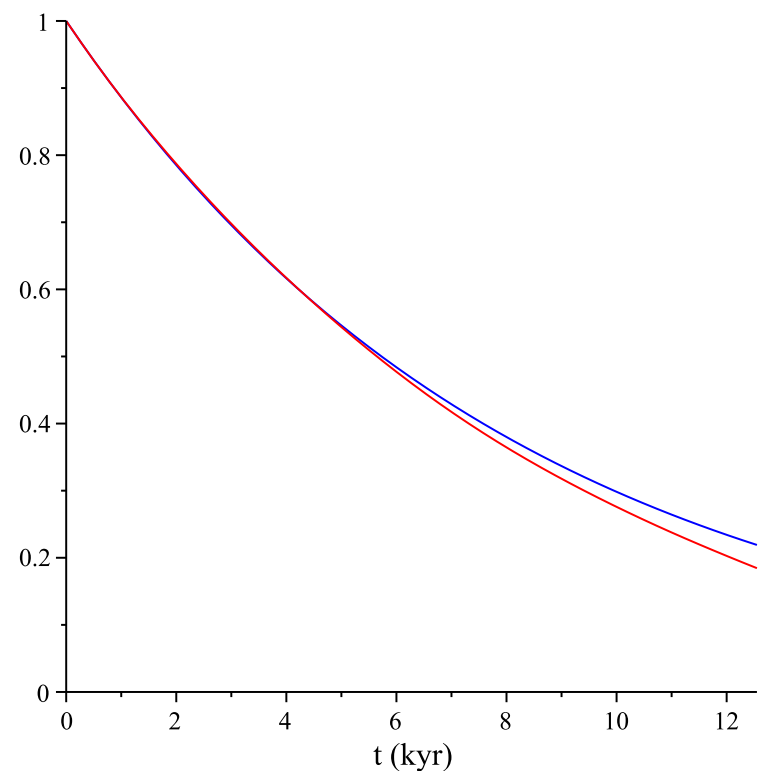

Figure 3: $y(t)$ given by (5) (red) compared with the exponential function $e^{-\alpha_{C} t}$ (blue) with $\alpha_{C}=\ln 2 / 5730 \mathrm{yr}^{-1}$.

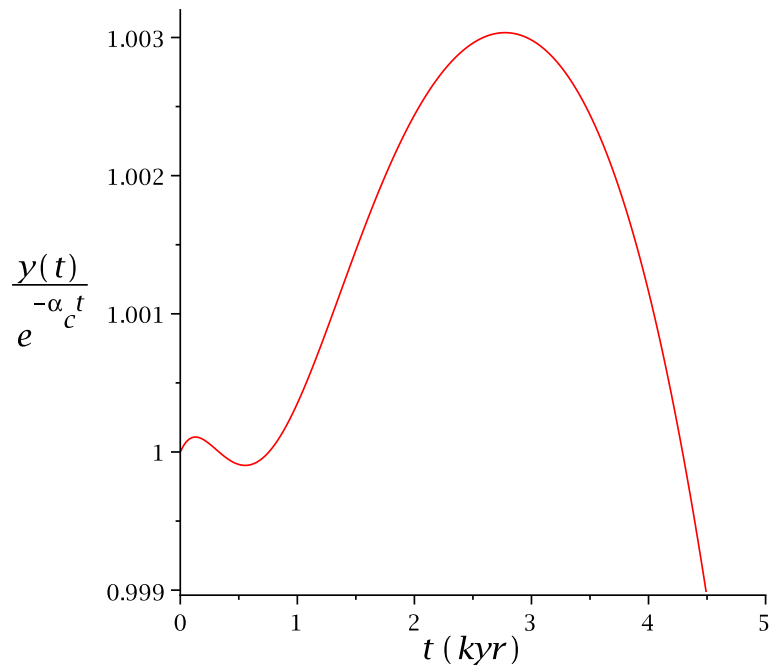

Figure 4: Plot of $y(t) / e^{-\alpha_{C} t}$ showing small amplitude oscillations.

Using AMS, both the ${ }^{14} \mathrm{C} /{ }^{13} \mathrm{C}$ and ${ }^{13} \mathrm{C} /{ }^{12} \mathrm{C}$ ratios of the sample and a standard are measured. The ${ }^{14} \mathrm{C} /{ }^{13} \mathrm{C}$ ratio is normalised to take account of isotopic fractionation, which adjusts the ${ }^{13} \mathrm{C} /{ }^{12} \mathrm{C}$ ratio of the sample to be the same as that of the standard, and an adjustment to the 1950 level is made. The normalised ${ }^{14} \mathrm{C} /{ }^{13} \mathrm{C}$ ratios of the sample and the standard are denoted by $(14 / 13)_{\mathrm{SN}}$ and $(14 / 13)_{\mathrm{ON}}$ respectively and the radiocarbon age $\tau_{r}(\mathrm{BP})$ is then given by [7]

$$
\tau_{r}=-\frac{1}{\alpha_{L}} \ln \left(\frac{(14 / 13)_{\mathrm{SN}}}{(14 / 13)_{\mathrm{ON}}}\right),
$$

where, by convention and for consistency with (33), the Libby half life is again used for the decay constant. Again assuming that the true decay curve for the ${ }^{14} \mathrm{C} /{ }^{12} \mathrm{C}$ ratio is given by $Y(t)$, then we have that $(14 / 13)_{\mathrm{SN}} /(14 / 13)_{\mathrm{ON}}=Y\left(t_{0}\right) / A_{0}$, where $t_{0}$ is the true age, since the 
${ }^{13} \mathrm{C} /{ }^{12} \mathrm{C}$ ratios of the sample and the standard are the same. Thus, equation (6) becomes

$$
\tau_{r}=-\frac{1}{\alpha_{L}} \ln \left(\frac{Y\left(t_{0}\right)}{A_{0}}\right)=-\frac{1}{\alpha_{L}} \ln y\left(t_{0}\right) .
$$

Now $t_{0}$ is the true age of the sample, which we denote by $t_{0}=\tau_{d}$, and so from equation (7) we now get a new calibration curve based on AMS measurements given by

$$
\tau_{r}=C_{\mathrm{AMS}}\left(\tau_{d}\right)=-\frac{1}{\alpha_{L}} \ln y\left(\tau_{d}\right),
$$

where the function $y$ is given by (5). This curve is shown in fig. 2, together with the line $\tau_{r}=\left(\alpha_{C} / \alpha_{L}\right) \tau_{d}$ which is associated with exponential decay. From this, we see that if assumption (ii) holds then AMS measurements should give consistently older radiocarbon dates than those obtained using radiometric methods for $\tau_{d}>3 \mathrm{cal} \mathrm{kBP}$.

While the predicted $\Delta^{14} \mathrm{C}$ curve which arises from case (i) cannot easily be verified experimentally, our prediction from case (ii), that radiocarbon dates obtained using the two different methods should be significantly different for older samples, is very easy to check using results of the Fourth International Radiocarbon Intercomparison (FIRI) [24]. These results show no significant difference in the predicted ages of a range of standard samples, including a sample of humic acid (sample E) dated at around $11.8 \mathrm{kBP}$, which is just beyond the range that we are considering. The AMS mean age for this sample was $54 \pm 53{ }^{14} \mathrm{C}$ yr higher than the corresponding age obtained using Gas Proportional Counting, a radiometric method, which is nowhere near the approximately $3,000{ }^{14} \mathrm{C}$ yr difference that our results predict for this sample.

These results would appear to invalidate the assumptions of case (ii) and support the conventional (but not verified) assumptions of case (i). However, before dismissing case (ii), we make two proposals that might explain why this predicted difference in dates is not observed.

Firstly, case (ii) is the extreme case. In the more likely scenario in which the discrepancy in the calibration curve is due to a combination of atmospheric variation and non-exponential decay the predicted difference between $C_{\mathrm{AMS}}$ and $C_{\beta}$ would be smaller.

Secondly, we note that radiometric methods were the first to be used for carbon dating. In the late 1970's, AMS techniques were developed to measure the ${ }^{14} \mathrm{C} /{ }^{12} \mathrm{C}$ ratio directly [25]. However, 'absolute AMS isotopic measurements are extremely difficult' [26] and so these methods were calibrated using international standards and modern samples of known age. For older samples, and given a strong belief in exponential decay, the results were also compared with the predicted radiocarbon ages obtained by radiometric methods. Indeed, the first aim of the FIRI exercise was 'demonstration of the comparability of routine analyses carried out by both AMS and radiometric laboratories' [24].

In practice, AMS measurements are the product of complex experimental procedures [25]. Results are affected by a number of factors, such as contamination during chemical processing [7, 27, 28], sample size [27, 29] and pretreatment of samples by acid hydrolysis [29, 24]. A small change in the AMS measurement as a result of any of these factors would be sufficient to generate a significant difference in the predicted radiocarbon age for older samples.

We therefore conclude that as long as AMS measurements are calibrated to give agreement with results from radiometric methods, our predicted difference in radiocarbon dates between the two methods will never be observed. 


\section{Conclusions}

The question posed in the title of this paper as to whether radioactive decay is really exponential (in all circumstances) is of fundamental importance, both theoretically and practically. We have presented experimental evidence, from the results of radiocarbon dating and modelling, which suggests that non-exponential decay may be a significant factor in the discrepancy in radiocarbon dates. We have also noted that quantum mechanics does not predict exact exponential decay, and have presented mathematical arguments that non-exponential decay should be expected for slowly decaying isotopes, but not for rapidly decaying isotopes. Thus, we believe that there is strong evidence, both experimental and theoretical, that radioactive decay of slowly decaying isotopes is not exactly exponential.

We have also shown that one significant consequence of non-exponential decay is that the two methods used for radiocarbon dating, namely radiometric and AMS, should give different results for older samples. This effect is not seen in practice, but we suggest that this could be due to AMS being calibrated to give agreement with results obtained using radiometric methods.

Our results in the previous section provide a way to experimentally test our prediction that the discrepancy in the calibration curve is due to a combination of atmospheric variation and non-exponential decay. To do this, a careful calibration of AMS measurements is required to ensure that correct absolute measurements are being made, particularly in the range 3-12.55 cal kBP. Dating of tree samples in this range should then be performed. If these radiocarbon dates are significantly higher than those in the INTCAL09 dataset, then it would confirm our prediction. Conversely, if good agreement is obtained with the INTCAL09 data, then it would disprove our prediction.

The question we are addressing of whether radioactive decay is exponential over long time periods is certainly of much theoretical interest. However, the stakes are high in more practical realms, since many dating methods over long time periods rely on the assumption of exponential decay of a slowly decaying isotope [4]. If the decay of ${ }^{14} \mathrm{C}$ is indeed non-exponential, then no other slowly decaying isotope can be assumed to decay exponentially either, which would remove a foundation stone of modern dating methods. If confirmed, this will require a radical reappraisal both of our approach to radioisotope dating methods and of the many currently accepted dates which have been obtained using these methods.

\section{Acknowledgements}

I am grateful to Professor Ron Johnson for many helpful discussions and comments and his continued interest in this work, to Professors Bill Gelletly, Jeff Tostevin and Phil Walker for commenting on an early draft and to Professor Michael Kearney for some helpful comments.

\section{References}

[1] Gopych, P. M., Zalyubovskii, I. I., Sotnikov, V. V., Shchus', A. F., Barchuk, I. F., Bulkin, V. S., Golyshkin, V. I. \& Ogorodnik, A. F. Study of the exponential nature of the decay law in the case of ${ }^{116 m_{1}}$ In. Sov. J. Nucl. Phys. 39, 159-160, 1984.

[2] Norman, E. B., Gazes, S. B., Crane, S. G. \& Bennett, D. A. Tests of the exponential decay law at short and long times. Phys. Rev. Letts 60, 2246-2249, 1988.

[3] Newton, R. G. Scattering theory of waves and particles. New York: McGraw-Hill, 1966. 
[4] Aitken, M. J. Science-based dating in archaeology. New York: Longman, 1990.

[5] Stuiver, M. \& Polach, H. A. Discussion: reporting of ${ }^{14} \mathrm{C}$ data. Radiocarbon 19, 355-363, 1977.

[6] Dicus, D. A. et al. Phys. Rev. A 65 032116, 2002.

[7] Donahue, D. J., Linick, T. W. \& Jull, A. J. T. Isotope-ratio and background corrections for accelerator mass spectrometry radiocarbon measurements. Radiocarbon 32, 135-142, 1990.

[8] Reimer, P. J., et al. InTCAL04 terrestrial radiocarbon age calibration, 0-26 cal kyr BP. Radiocarbon 46, 1029-1058, 2004.

[9] Friedrich, M., Remmele, S., Kromer, B., Hofmann, J., Spurk, M., Kaiser, K. F., Orcel, C. \& Küppers, M. The 12,460-year Hohenheim oak and pine tree-ring chronology from central Europe - a unique annual record for radiocarbon calibration and paleoenvironment reconstructions. Radiocarbon 46, 1111-1122, 2004.

[10] Schaub, M., Kaiser, K. F., Frank, D. C., Büntgen, U., Kromer, B. \& Talamo, S. Environmental change during the Allerød and Younger Dryas reconstructed from Swiss tree-ring data. Boreas 37, 74-86, 2008.

[11] Reimer, P. J. et al. IntCAL09 and MARINE09 radiocarbon age calibration curves, 0-50,000 years cal BP. Radiocarbon 51, 1111-1150, 2009.

[12] Bard, E. Geochemical and geophysical implications of the radiocarbon calibration. Geochim. Cosmo. Acta 62, 2025-2038, 1998.

[13] Beck, J. W., et al. Extremely large variations of atmospheric ${ }^{14} \mathrm{C}$ concentration during the last glacial period. Science 292, 2453-2458, 2001.

[14] Stuiver, M. et al. InTCAL98 radiocarbon age calibration, 24,000-0 cal BP. Radiocarbon 40, 1041-1083, 1998.

[15] Hughen, K., Lehman, S., Southon, J., Overpeck, J., Marchal, O., Herring, C. \& Turnbull, J. ${ }^{14} \mathrm{C}$ activity and global carbon cycle changes over the past 50,000 years. Science 303, 202-207, 2004.

[16] Chiu, T. C., Fairbanks, R. G., Cao, L. \& Mortlock, R. A. Analysis of the atmospheric ${ }^{14} \mathrm{C}$ record spanning the past 50,000 years derived from high-precision ${ }^{230} \mathrm{Th} /{ }^{234} \mathrm{U} /{ }^{238} \mathrm{U}$ ${ }^{231} \mathrm{~Pa} /{ }^{235} \mathrm{U}$ and ${ }^{14} \mathrm{C}$ dates on fossil corals. Quat. Sci. Revs 26, 18-36, 2007.

[17] Roberts, M. L. \& Southon, J. R. A preliminary determination of the absolute ${ }^{14} \mathrm{C} /{ }^{12} \mathrm{C}$ ratio of OX-I. Radiocarbon 49, 441-445, 2007.

[18] Evans, R. D. The atomic nucleus, 11th edn. New York: McGraw-Hill, 1967.

[19] Fonda, L., Ghirardi, G. C. \& Rimini, A. Decay theory of unstable quantum systems. Rep. Prog. Phys. 41, 587-631, 1978.

[20] Merzbacher, E. Quantum mechanics, 3rd edn. New York: Wiley, 1998.

[21] Peres, A. Nonexponential decay law. Ann. Phys. 129, 33-46, 1980. 
[22] Stewart, I. Does God play dice? Oxford: Blackwell Publishing, 2002.

[23] Stuiver, M. Variations in radiocarbon concentration and sunspot activity. J. Geophys. Res. 66, 273-276, 2002.

[24] Scott, E. M. (ed.) The Fourth International Radiocarbon Intercomparison (FIRI). Radiocarbon 45(2), 2003.

[25] Fifield, L. K. Accelerator mass spectrometry and its applications. Rep. Prog. Phys. 62, 1223-1274, 1999.

[26] Nishiizumi, K., Imamura, M., Caffee, M. W., Southon, J. R., Finkel, R. C. \& McAninch, J. Absolute calibration of ${ }^{10}$ Be AMS standards. Nucl. Inst. Meth. Phys. Res. B 258, 403-413, 2007.

[27] Brown, T. A. \& Southon, J. R. Corrections for contamination background in AMS ${ }^{14} \mathrm{C}$ measurements. Nucl. Inst. Meth. Phys. Res. B 123, 208-213, 1997.

[28] Mueller, K. \& Muzikar, P. Correcting for contamination in AMS ${ }^{14} \mathrm{C}$ dating. Radiocarbon 44, 591-595, 2002.

[29] Brown, L., Cook, G. T., MacKenzie, A. B. \& Thomson, J. Radiocarbon age profiles and size dependency of mixing in northeast Atlantic sediments. Radiocarbon 43, 929-937, 2001. 